2013-06-01

\title{
Numerical and physical modelling of extreme waves at Wave Hub
}

\author{
Ransley, E::0000-0003-1446-7907
}

http://hdl.handle.net/10026.1/13629

10.2112/SI65-278.1

Journal of Coastal Research, Special Issue

Coastal Education and Research Foundation

All content in PEARL is protected by copyright law. Author manuscripts are made available in accordance with publisher policies. Please cite only the published version using the details provided on the item record or document. In the absence of an open licence (e.g. Creative Commons), permissions for further reuse of content should be sought from the publisher or author. 


\title{
Numerical and physical modeling of extreme waves at Wave Hub
}

\author{
Edward Ransley, Martyn Hann, Deborah Greaves, Alison Raby, Dave Simmonds \\ School of Marine Science and \\ Engineering, University of Plymouth, \\ Plymouth, PL4 8AA, UK \\ edward.ransley@plymouth.ac.uk \\ martyn.hann@plymouth.ac.uk \\ deborah.greaves@plymouth.ac.uk \\ alison.raby@plymouth.ac.uk \\ d.simmonds@plymouth.ac.uk
}

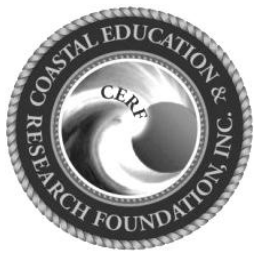

www.cerf-jcr.org

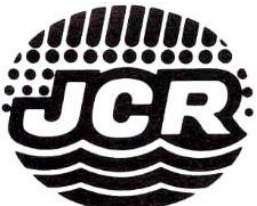

www.JCRonline.org

\section{ABSTRACT}

Ransley, E., Hann, M., Greaves, D., Raby, A. and Simmonds, D., 2013. Numerical and physical modelling of extreme waves at Wave Hub In: Conley, D.C., Masselink, G., Russell, P.E. and O'Hare, T.J. (eds.), Proceedings $12^{\text {th }}$ International Coastal Symposium (Plymouth, England), Journal of Coastal Research, Special Issue No. 65, pp. 16451650, ISSN 0749-0208.

\begin{abstract}
With a history of international failures, the survivability of coupled systems of wave energy devices and their moorings, particularly those to be installed at development sites like Wave Hub, is surrounded by uncertainty. Potential design solutions require a better understanding of the hydrodynamics and structural loading experienced during extreme events, like rogue wave impact, in order to mitigate the risk of device and mooring failure. Rogue waves are waves with amplitudes far greater than those expected, given the surrounding sea conditions. Intense study into these events stems from their potential for catastrophic impact on ocean engineering structures. However, little is known about their physical origins and, currently, there is no consensus on their definition or explanation of the mechanism which drives them. This paper concerns the numerical modeling and experimental validation of extreme rogue wave examples at the Wave Hub site. Using hindcast data, the 100 year extreme wave at the Wave Hub site is determined. This extreme wave is replicated in Plymouth University's new COAST Lab using a NewWave, dispersive focusing input. To simulate and analyse these events, we duplicate these conditions in a numerical wave tank (NWT), solving the fully nonlinear Navier-Stokes equations, with a free surface, using the Volume of Fluid (VoF) method and open source CFD library OpenFOAM ${ }^{\circledR}$. The comparison shows that the CFD software is capable of simulating focused waves similar to those produced in the physical tank but tends to overestimate the crest heights. It is also noted that nonlinear effects are important when considering the shape and location of focused wave events.
\end{abstract}

ADDITIONAL INDEX WORDS: Wave energy, WEC design, survivability, nonlinear waves, NewWave, OpenFOAM®, waves 2 Foam.

\section{INTRODUCTION}

The undeniable changes to the climate system and the steady increase in global energy demand, through population growth and improved living conditions, are now major concerns for many of the world's governments. The ratification of the Kyoto protocol marked a step change in government attitudes towards $\mathrm{CO}_{2}$-free energy sources. In many countries the focus has now switched in favour of renewable energy sources to meet emissions targets, secure cost-effective energy supplies and prevent further anthropogenic interference with the Earth's climate system. Until recently, the search for economical, renewable and sustainable solutions to electricity production, in many countries, has been centred on wind, solar and biomass (Leijon et al., 2003).

Even though wave energy represents a renewable source with high power-density, relatively high utilization factor and few negative impacts, extracting energy from ocean waves has been considered uneconomical due to a lack of good engineering solutions (Leijon et al., 2006, Henfridsson et al., 2007). If, however, economical and technical solutions were developed, the area would have a vast impact on the electricity production in the world, particularly in countries with significant resource like the United Kingdom.

In light of this, an increasing number of government funded test sites for pre-commercial wave energy converters (WECs), are being developed throughout the World and a number of full-scale

DOI: 10.2112/SI65-278.1 received 07 December 2012; accepted 06 March 2013.

(C) Coastal Education \& Research Foundation 2013 individual WECs have now been successfully operated at sea. Wave Hub is a $20 \mathrm{MW}$ wave farm currently under development in the UK. Its aim is to provide a link between the testing of fullscale devices and the commercial deployment of wave energy arrays (Smith et al., 2012). However, a history of international WEC and mooring failures demonstrates that considerable uncertainty exists around the prototype behaviour of fully coupled dynamic systems comprising WECs and their moorings.

Research directly concerning WECs has focussed primarily on optimal response. In a design context, this is not always desirable (as highlighted by the recent reliability issues) and there appears to be a lack of research into the survivability of WECs with the exception of conceptual criteria proposed by Ocean Power Delivery Ltd (OPD), who have been developing the Pelamis concept. OPD prioritise survivability over power capture ensuring devices feature inherent load shedding and de-tuning capabilities in large waves (Yemm et al., 2012).

In terms of survivability, it is the sometimes catastrophic impacts from abnormally large, 'freak' or 'rogue', waves which are of most concern to WEC developers. These 'extreme' waves, with amplitudes far exceeding the traditional design parameters for a particular wave field, have received significant attention since 2000 after a few surprising offshore observations. The most notable of which was the Draupner or 'New year' wave which struck the Draupner oil platform in the North Sea on the 1st of January 1995. Extreme waves are very steep and highly-nonlinear, characterised by a highly distorted free surface and overturning, and can have up to ten times the average energy of waves in that 
sea state (Dysthe et al., 2009). However, despite intensive study, there is no generally accepted theory or explanation for their occurrence and no consensus on how to define their shape.

It is well known that unexpected, large waves can form through refraction in coastal waters or areas with strong currents but there is some debate over how extreme waves are produced in the deep ocean. Some of the most important mechanisms proposed, in order to explain the existence of extreme waves, are as follows: Superposition is typical of the standard linear model and extreme waves have been recorded in standard irregular seas where several waves sum together in phase. This is only slightly altered at 2 nd order but at higher orders the interactions between waves become important; spatial focussing is caused by refraction of waves by bathymetry or currents and can lead to crest alignment or trapping of waves; dispersive focussing is another linear effect taking into account the frequency dependence of wave celerity. A chirped wave packet can be formed by adding waves of different frequencies in such a way that they combine constructively at a specific point in space and time. Chirped wave trains can exhibit strong focussing even if a random wave field is also present, however there is some controversy over whether or not these contrived wave forms are to be the likely cause of real extreme waves (Dysthe et al., 2009); nonlinear focussing has also been proposed including the application of the nonlinear Schrödinger equation (NSL) (from quantum physics) and the modulation instability of a regular unidirectional wave train known as the Benjamin-Feir instability (Clauss, 2010). However, although the concept is physically and mathematically robust, it remains unclear as to whether or not the phenomenon can be practically responsible for extreme waves in realistic ocean conditions (Dysthe et al., 2009).

In the context of wave energy development, both physical and numerical modelling have become increasingly important in the assessment of a given concept before going to the expense of full scale deployment at sea. Developers consider this type of testing to be a vital stage in the engineering development of WECs to ensure the device behaves as expected and no unexpected and costly damage is caused when deployed.

Physical modelling of wave structure interactions using scale model WECs in a wave tank has been widely used by developers. However, complicated experiments do not come without an associated cost in both terms of time and capital and in many cases the required analysis is very difficult, or impossible, as some quantities maybe immeasurable without disturbing the flow itself.

The continued increase in the performance-to-cost ratio of modern computers has meant that numerical models can now offer a means to interpret the fundamental phenomenological aspects of experimental conditions that physical tests may not. However, without validation from physical tests, the consequences of accepting such a result may be severe (Ferziger and Perić, 2002). As part of the wider SuperGen UK Centre for Marine Energy Research (UKCMER) project, led by the present authors, entitled 'Survivability of Wave Energy Converter and Mooring coupled system', the aim of this research is to develop a robust combination of, and improve convergence between, experimental measurement techniques and numerical modelling approaches for extreme wave impacts. The present paper provides a description of the physical and numerical simulations completed, including the experimental set-up, focussed wave input (using the NewWave theory) and the computational fluid dynamics (CFD) software used, OpenFOAM ${ }^{\circ}$. Extreme wave conditions characteristic of the Wave Hub site are simulated in both environments and a comparison drawn between the results. This provides an insight into the flow conditions around extreme wave events and helps to develop a numerical tool that can yield a reliable understanding of extreme loading conditions, enhancing the design, development and operational efficiency of WEC technologies.

\section{EXPERIMENTAL METHOD}

\section{Wave Hub and NewWave Input}

Wave Hub is a designated wave energy array test site, located $16 \mathrm{~km}$ off the north coast of Cornwall, south west UK. Water depths are generally 50 to 60 meters across the site. Wave data has been collected since 2005 and is publically available (JP Kenny, 2009). Sets of modeled wave data are also available from the UK Met Office for longer periods of time. It is from these data sets that waves with a 1 in 100 year return period at Wave Hub have been derived using a Weibull fit (SWERDA, 2006) giving $H_{s}=$ $14.4 \mathrm{~m}$ and $T_{z}=14.1 \mathrm{~s}$. Here, $H_{s}$ is the significant wave height defined as the mean height of the highest third of the waves measured. $T_{z}$ is the mean zero up-crossing period.

A random wave based around these statistics could be used to predict the interaction of a wave energy device with the 100 year event. These however require long term simulation to generate extreme events. An alternative approach, used frequently by the offshore industry as a design wave, is the NewWave formulation introduced by Tromans et al. (1991). This compact wave packet has a local time history identical to the scaled autocorrelation function (the Fourier transform of the spectrum of the sea state) (Hunt-Raby et al., 2011). The concept of the NewWave theory is to generate an extreme wave, at a known position and time, through the superposition of small amplitude linear waves of varying periods. For large crests, the most probable values of water elevation around the crest can then be generated in a reproducible way.

For a linear crest focused wave group the surface elevation is

$$
\eta(x, t)=\sum_{n} a_{n} \cos \left(k_{n} x-\omega_{n} t-k_{n} x_{f}+\omega_{n} t_{f}\right) x
$$

where $x$ is distance, $t$ is time, $n$ identifies the frequency component, $k_{n}$ is wave number, $\omega_{n}$ is angular frequency, $a_{n}$ is wave amplitude, $x_{f}$ is focus point and $t_{f}$ is focus time. For NewWave the amplitudes of the individual components are calculated by

$$
a_{n}=\frac{A S_{n}(\omega) \Delta \omega_{n}}{\sum_{n} S_{n}(\omega) \Delta \omega_{n}}
$$

where $S_{n}(\omega)$ is the energy spectrum, $\Delta \omega_{n}$ is the frequency increment and $A$ is the crest amplitude. By setting the crest amplitude to

$$
A=\sqrt{2 m_{0}(\ln (N))}
$$

the NewWave models the largest wave in $N$ waves.

The wave climate at Wave Hub is considered to be fetch limited (SWERDA, 2006), and therefore a JONSWAP spectrum was assumed when applying the NewWave theory

$$
\left.S(f)=\left(\frac{f_{p}}{f}\right)^{5} \exp \left(-\frac{5}{4}\left(f_{p} / f\right)^{4} \gamma^{\exp \left(\frac{-\left(f-f_{p}\right)^{2}}{2 \sigma^{2} f_{p}^{2}}\right.}\right)\right)
$$

where $f_{p}$ is peak frequency and $\sigma=0.07 f<f_{p} \sigma=0.09 f \geq f_{p}$ and $\gamma$ $=3.3$, as commonly used in the offshore industry. A 3 hour sea 


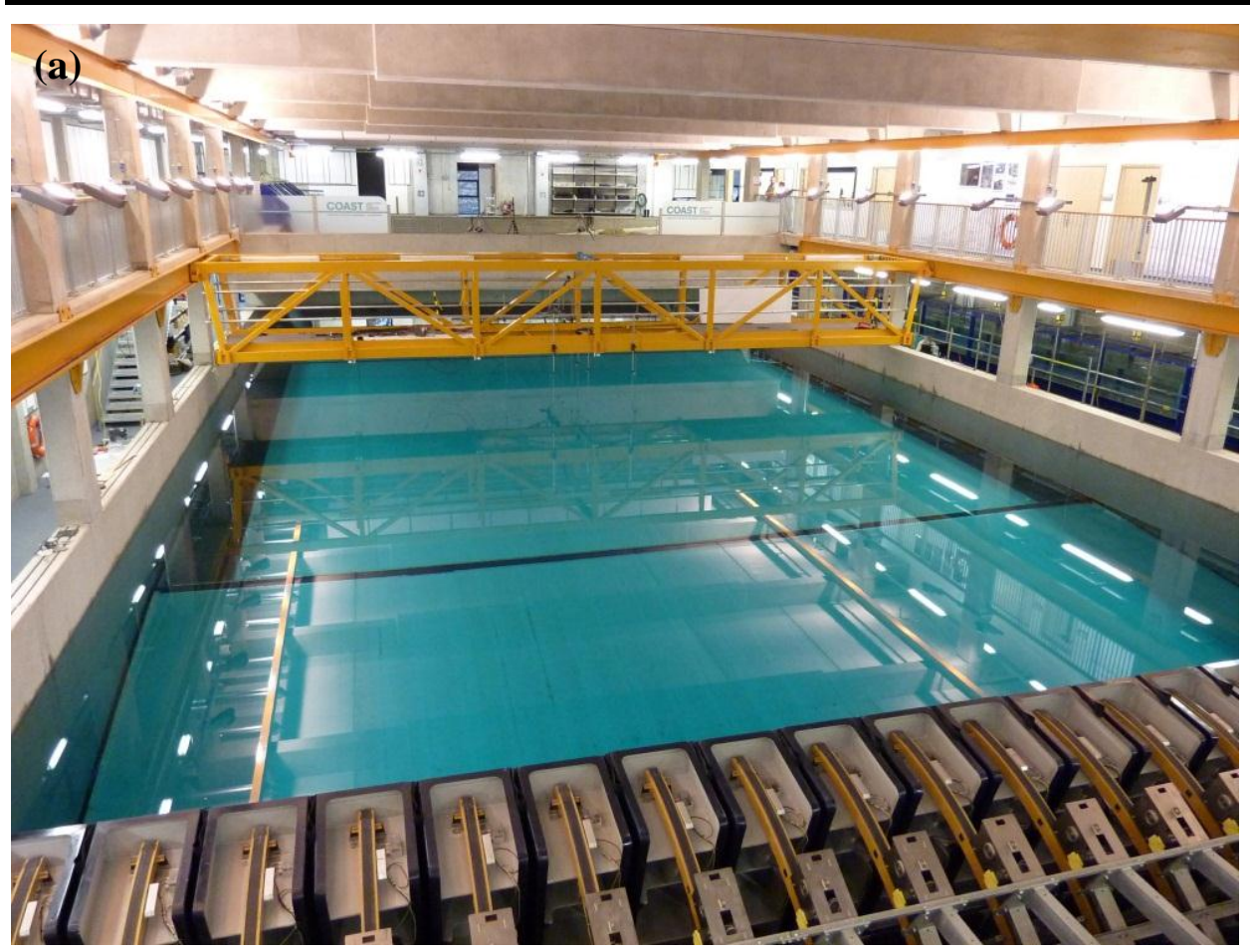

(b)

\begin{tabular}{|c|c|}
\hline $\begin{array}{c}\text { Wave } \\
\text { Gauge }\end{array}$ & $\begin{array}{c}\text { Distance from } \\
\text { wave paddle }(\mathrm{m})\end{array}$ \\
\hline G1 & 18.57 \\
\hline G2 & 19.05 \\
\hline G3 & 19.56 \\
\hline G4 & 20.01 \\
\hline G5 & 20.50 \\
\hline G6 & 20.82 \\
\hline G7 & 21.12 \\
\hline G8 & 21.61 \\
\hline
\end{tabular}

Figure 1. (a) The COAST Lab Ocean basin at Plymouth University. (b) Table of wave probe positions used in the experimental set-up.

state is assumed to have approximately 1000 waves (Hunt-Raby et al., 2011) and therefore $\mathrm{N}=1000$.

\section{Experimental Set-Up}

The 100 year return period NewWave group was generated at $50^{\text {th }}$ scale in the new Plymouth University COAST laboratory Ocean basin. This is a $35 \mathrm{~m} \times 15.5 \mathrm{~m}$ tank with a variable floor depth (maximum depth of $3.0 \mathrm{~m}$ ) and 24 flap type wave paddles (Figure 1(a)). A floor depth of $2.8 \mathrm{~m}$ was used. Although not correctly scaled to the Wave Hub site, this deep-water regime was used to limit the nonlinear floor effects for these initial tests. Measurements using a correctly scaled intermediate depth regime of $1.5 \mathrm{~m}$ are planned. Waves were measured using eight resistive wave gauges, mounted along the center of the tank, in the locations shown in Figure 1(b).

243 wave components with frequencies evenly spaced between 0 and $2 \mathrm{~Hz}$, were produced from the NewWave theory described above, to create a focused wave located at the paddles at time $=$ 0 s. The wave paddle control software was then used to shift this focused wave to the required theoretical focus point and time.

A wave group is defined as focused when all components are completely in phase. This can be judged by eye to occur where the troughs either side of the central peak are the same size (Raby, 2003). Due to non-linear wave-wave interactions the linear theoretical focus and the distance from the paddles at which the actual focus occurred were different. A trial and error approach was used to focus the wave group at the $6^{\text {th }}$ probe in the sequence, $20.82 \mathrm{~m}$ from the wave paddles, by adjusting the theoretical focus to $19.6 \mathrm{~m}$.

\section{NUMERICAL METHOD}

Numerical simulation can provide a valuable insight into the processes behind extreme events and generate crucial data needed for informed design parameters. There is an extensive literature concerning the numerical modelling of fluid mechanics and it would not be feasible to cover it all in this paper. Furthermore, the range of scales considered for simulation is vast, with methods and outputs typically scale specific. This project is concerned with the single episodic event of an extreme wave impact on a single WEC, the nonlinear coupling between the WEC, power take-off (PTO) and mooring system and the implications this has on the survivability of the device. Therefore only suitable methods for this problem are considered and those methods which are widely considered inappropriate for nonlinear interactions at the device scale have been ignored.

\section{Free Surface Modeling Techniques}

The case of extreme wave interactions with coupled WEC and mooring systems requires a fully nonlinear, time-domain solution. The method used must be able to accommodate a highly distorted, multivalued free-surface and cope with topological changes like wave breaking and recombination in 3D. Accurate simulation of a moving fluid interface, particularly one which is highly distorted, is extremely challenging. The position of the interface must be calculated as part of the solution as it is not known in advance of any given time-step (Greaves, 2004). The few strategies appropriate for free-surface modeling can be divided into two categories: surface tracking schemes including nonlinear potential flow and particle tracking methods, and; surface capturing schemes including, Navier-Stokes solvers like the Volume of Fluid (VoF) and Level Set (LS) methods (Gao et al., 2007).

Surface (or interface) tracking schemes solve for the flow in the fluid region only. The free-surface is modeled as a moving boundary containing the fluid domain. The instantaneous position of the boundary is defined by applying a kinetic boundary condition. This allows for the location of the free-surface to be calculated precisely, however, the mesh-based methods require 


\begin{tabular}{|c|c|c|c|}
\hline$\longleftarrow$ Inlet Boundary & $1 m$ & Air & \\
\hline $30 \mathrm{~m}$ & $2.8 \mathrm{~m}$ & Water & $5 m$ \\
\hline
\end{tabular}

Figure 2. Computational domain of numerical simulations.

constant repositioning of the grid points introducing numerical errors and a low stability (Gao et al., 2007). Furthermore, meshbased surface tracking methods do not perform well when there are high levels of surface distortion like wave breaking as they cannot accommodate a multivalued interface and typically ignore the effects of viscosity and turbulence. Particle tracking methods, like smooth particle hydrodynamics (SPH), are very powerful, eliminating the need for a mesh and have been gaining popularity recently (Stansby et al., 2008), but, these methods are still considered to be too computationally demanding and therefore unpractical in three dimensions (Greaves, 2004).

In surface (or interface) capturing schemes both fluids are solved on a fixed grid and a marker function is used to identify the position of the interface, where a discontinuity in the density field exists, eliminating the need for specialised free-surface tracking. In these methods the full Navier-Stokes equations can be solved with an additional transport equation, for the particular marker function, solved at each time step to reconstruct the free-surface. These methods are more robust than surface tracking methods and can include viscous effects, but capturing and reconstructing the interface is complicated and comes at a high CPU cost (Gao et al., 2007). There are a number of different surface capturing methods, each distinguishable by the individual marker function used. For example, in the Marker And Cell method (MAC) marker particles are utilised, in the Volume of Fluid ( $\mathrm{VoF}$ ) method a volume fraction field is used and in the Level Set (LS) method the interface is located via the zero contour of a distance function. Both the VoF and LS methods are known as front capturing methods and have been shown to be capable of simulating large scale deformations of the free-surface including wave breaking and merging (Greaves, 2004).

\section{OpenFOAM $®$ and the Waves2Foam Toolbox}

The Open Source Field Operation and Manipulation C++ Library (OpenFOAM $\AA$ ) is a freely available set of applications developed to solve particular problems in continuum mechanics. Being open source, OpenFOAM $\AA$ is gaining popularity in coastal engineering studies where large computational demands, requiring parallel processing, are common place and typically expensive to run on commercial platforms (Jacobsen et al., 2011).

For the case of free surface Newtonian flows, OpenFOAM® contains a solver, called interFoam, for solving the Reynolds averaged Navier-Stokes (RANS) equations for the combined flow of air and water,

$$
\begin{gathered}
\frac{\partial \rho \mathbf{V}}{\partial t}+\nabla \cdot\left[\rho \mathbf{V V} V^{T}\right]=-\nabla p^{*}-\mathbf{g} \cdot \mathbf{x} \nabla \rho+\nabla \cdot[\mu \nabla \mathbf{V}+\rho \tau] \\
+\sigma_{T} K_{\alpha} \nabla \alpha
\end{gathered}
$$

coupled with the incompressible continuity equation

$$
\nabla \cdot \mathbf{u}=0 .
$$

where, $\mathbf{V}=(u, v, w)$ is the velocity vector, $p^{*}$ is the pressure above hydrostatic pressure, $\rho$ is the density, $\mathbf{g}$ is the gravitational acceleration, $\mu$ is the dynamic molecular viscosity and $\tau$ is the specific Reynolds stress tensor. The final term in Equation (5), including the surface tension coefficient, $\sigma_{T}$, and the surface curvature, $\kappa_{\alpha}$, will have only minor effects in civil engineering applications (Jacobsen et al., 2011).

These equations are solved simultaneously for the two immiscible fluids using the finite volume method of discretization and a VoF approach similar to the formulation of Hirt and Nichols (1981). The pressure-velocity coupling is achieved through the PISO algorithm and the interface is captured using the scalar field $\alpha$ (which equals 0 for air and 1 for water) with a compression term to limit the interface smearing. The distribution of $\alpha$ is modeled by the advection equation

$$
\frac{\partial \alpha}{\partial t}+\nabla \cdot[\mathbf{V} \alpha]+\nabla \cdot\left[\mathbf{V}_{r} \alpha(1-\alpha)\right]=0
$$

(Jacobsen et al., 2011).

A crucial tool in coastal, offshore and maritime engineering applications, which is currently missing from the official distribution of OpenFOAM ${ }^{\circ}$, is the ability to generate free surface waves and absorb internally generated waves in a

convenient manner (Jacobsen et al., 2011). These two elements have received some attention in studies using OpenFOAM $®$ : Morgan et al. (2010) investigated the simulation of propagating water waves in OpenFOAM $\AA$, but did not include an outlet absorption method; (Afshar, 2010) developed an absorption method, although it was reliant on a highly refined mesh at the surface. Jacobsen et al. (2011) have since addressed and solved these two limitations developing a wave generation toolbox, named waves2Foam, which has the ability to be coupled with the standard $\mathrm{VoF}$ method within OpenFOAM ${ }^{\circledR}$ offering generic wave generation and an absorption scheme termed 'wave relaxation zones'. It has been shown that waves2Foam coupled with OpenFOAM ${ }^{\circledR}$ can accurately model the propagation and breaking of water waves (Jacobsen et al., 2011).

\section{Numerical Simulation Procedure}

In order to analyse the ability of the software and compare numerical results, generated using OpenFOAM $®$, with the experimental results obtained, a two-dimensional numerical wave tank (NWT) was constructed to mimic conditions in the physical tank. The NWT was $2.8 \mathrm{~m}$ deep and $30 \mathrm{~m}$ long with an additional $5 \mathrm{~m}$ relaxation zone opposite the inlet boundary (Figure 2). A uniform, square-celled grid was used to discretize the computational domain with $18225(135 \times 135)$ cells per $\mathrm{m}^{2}$. The water and air had densities of $1000 \mathrm{kgm}^{-3}$ and $1 \mathrm{kgm}^{-3}$; and, kinematic viscosities of $1 \times 10-6 \mathrm{kgm}^{-1} \mathrm{~s}^{-1}$ and $1.48 \times 10^{-5} \mathrm{kgm}^{-1} \mathrm{~s}^{-1}$ respectively. No turbulence model was used.

The waveFoam solver (a version of the interFoam solver coupled with the waves2Foam libraries) was used to model the free-surface flow using the same NewWave combination of $1^{\text {st }}$ order Stokes waves, as in the physical tests, to define the surface elevation and fluid velocities on the inlet boundary. Implicit Euler time stepping was used and the adjustableTimeStep function within OpenFOAM ${ }^{\circledR}$ utilized to satisfy the Courant-FriedrichsLewy (CFL) condition (important for stability when solving hyperbolic functions (Anderson, 1995)). Simulations were run in parallel on seven Intel ${ }^{\circledR}$ Xeon ${ }^{\circledR}$ E5430 processors at $2.66 \mathrm{GHz}$ each, using Ubuntu 10.04 (lucid) Linux kernel 2.6.32-44-generic and OpenFOAM ${ }^{\circledR}$ version 2.0.1. Full domain results were recorded every $0.5 \mathrm{~s}$ 


\section{RESULTS}

Figure 3(a) shows a time series of the surface elevation (normalised by the theoretical crest height) recorded during the physical experiment at the $6^{\text {th }}$ wave probe $(20.82 \mathrm{~m}$ from the wave paddle) and the results from the numerical investigation at the same location $(20.82 \mathrm{~m}$ from the inlet boundary).

Figure 3(b) shows a snapshot of the normalised free-surface, from the numerical simulation, for a range of times spread across the focus event. The surface beyond $30 \mathrm{~m}$ can be ignored as this region is within the numerical relaxation zone where the wave energy is being absorbed to remove reflections.

\section{DISCUSSION}

As can be seen from Figure 3(a), the numerical simulation has been reasonably successful in reproducing the physical conditions generated in the wave tank. The shallow troughs either side of the focussed crest have been modelled accurately and there is very little phase discrepancy between the results. The most notable difference is that the numerical solution appears to overestimate the height of the peaks (the numerical focused crest height is $21.1 \%$ higher than the experiment). This discrepancy is not unusual for preliminary experiments of this kind which can be affected strongly by the nature of the inlet boundary definitions on a case specific basis (Westphalen, 2008). In addition to this, there is some loss of symmetry about the crest but this may be due to a further shift in the focus location in the NWT just as the physical focus point is shifted when compared to the linear theory. By observing Figure 3(b) it can be seen that the true focus event in the NWT is between $30 \mathrm{~s}$ and $30.5 \mathrm{~s}$ with a crest location closer to $21.5 \mathrm{~m}$ (instead of $20.82 \mathrm{~m}$ ). These observations suggest that, with further calibration of this preliminary NWT, the physical extreme wave event may be reproduced with a good degree of accuracy using this method.
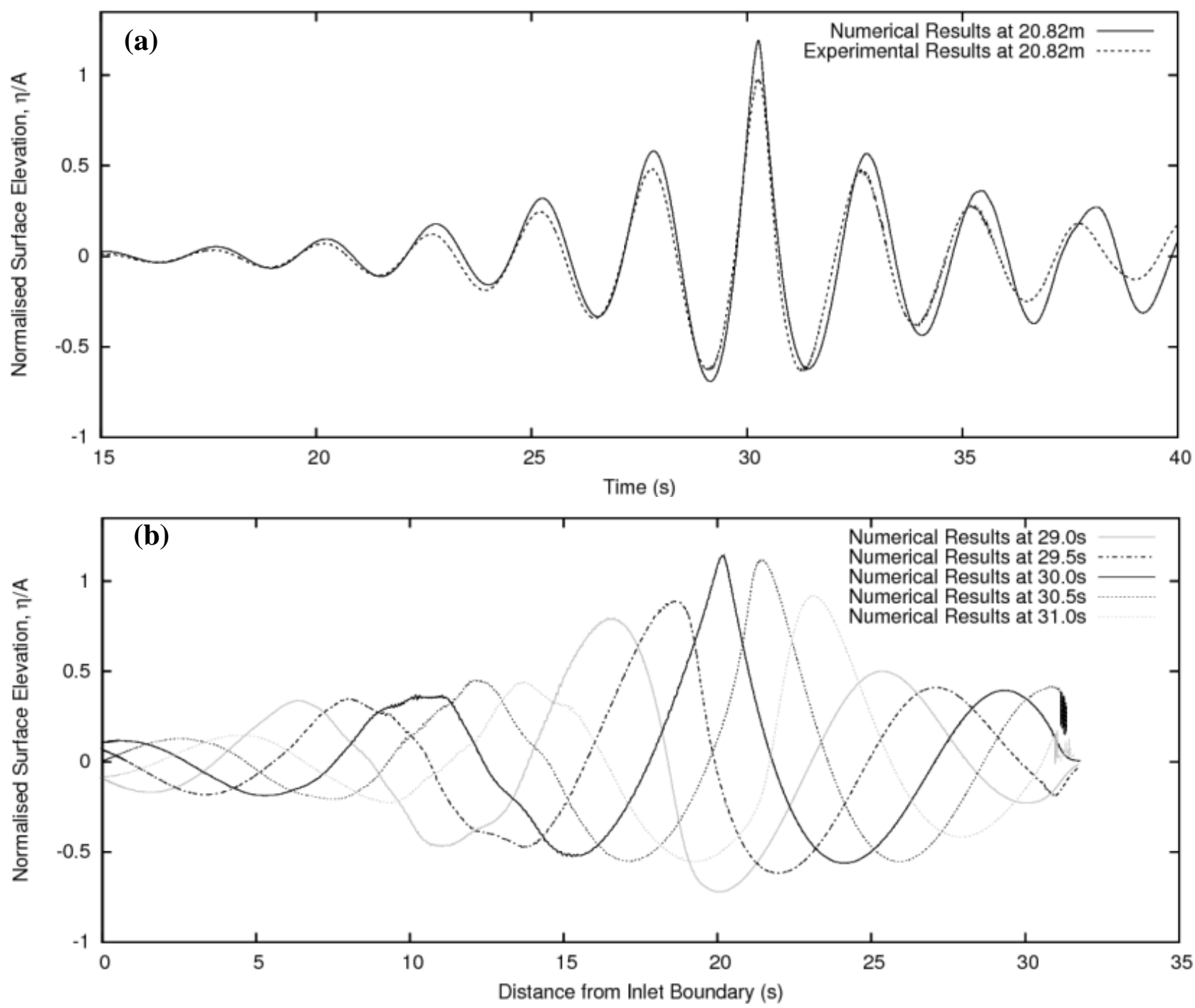

Figure 3. (a) Time series of the normalised surface elevation recorded for the physical (dashed) and numerical (solid) experiments at the physical focus location $(x=20.82 \mathrm{~m})$. (b) Free surface snapshot from the numerical simulations at five different times spaced evenly across the focus event. 


\section{CONCLUSIONS}

In conclusion, the OpenFOAM ${ }^{\circledR}$ and waves2Foam toolboxes are able to simulate a NewWave extreme wave event comparable to physical results for the same, reproducible, linear input. It has been demonstrated for an extreme 100 year wave event at the Wave Hub site showing that this software is suitable for the simulation of extreme waves. Further calibration will be undertaken and additional trials, using a time-series input taken directly from the physical tank, will be used to ensure the validity of the numerical model. The next phase of the project will then be to investigate the extreme wave loading on a floating structure.

\section{ACKNOWLEDGEMENTS}

The work described in this publication was supported by EPSRC SuperGen UKCMER through $\mathrm{PhD}$ studentship and the XMED project.

\section{LITERATURE CITED}

Anderson, J. J. D., 1995. Computational Fluid Dynamics: The Basics with Applications, New York, NY: McGraw-Hill International Editions.

Afshar, M. A. 2010. Numerical wave generation in OpenFOAM®. Gothenburg, Sweden: Chalmars University of Technology, Master's thesis.

Clauss, G. F., 2010. Freak Waves and Their Interaction with Ships and Offshore Structures. In: Ma, Q. (ed.), Advances in Numerical Simulation of Nonlinear Water Waves. World Scientific Publishing Co. Pte. Ltd, pp.641-687.

Dysthe, K., Krogstad, H. E. and Müller, P., 2009. Rogue waves. In: Steele, J. H., Turekian, K. K. and Thorpe, S. A. (eds.), Encyclopedia of Ocean Sciences (Second Edition). Oxford: Academic Press, pp.770-780.

Ferziger, J. H. and Perić, M., 2002. Computational Methods for Fluid Dynamics (3rd Edition), Verlag Berlin Heidelberg New York: Springer.

Gao, F., Ingram, D. M., Causon, D. M. and Mingham, C. G., 2007. The development of a Cartesian cut cell method for incompressible viscous flows. International Journal for Numerical Methods in Fluids, 54, 1033-1053.

Greaves, D. M. 2004. A quadtree adaptive method for simulating fluid flows with moving interfaces. Journal of Computational Physics, 194, 35-56.

Henfridsson, U., Neimane, V., Strand, K., Kapper, R., Bernhoff, H., Danielsson, O., Leijon, M., Sundberg, J., Thorburn, K., Ericsson, E. and Bergman, K., 2007. Wave energy potential in the Baltic Sea and the Danish part of the North Sea, with reflections on the Skagerrak. Renewable Energy, 32, 2069-2084.

Hirt, C. W. and Nichols, B. D., 1981. Volume of fluid (VOF) method for the dynamics of free boundaries. Journal of Computational Physics, 39, 201-225.

Hunt-Raby, A.C., Borthwick, A.G.L., Stansby, P.K. and Taylor R.H., 2011. Experimental measurements of focused wave group and solitary wave overtopping. Journal of Hydraulic Research, 49(4), 450-464.

Hunt, A., 2003. Extreme Waves, Overtopping and Flooding at Sea Defences. Oxford, UK: University of Oxford, Ph.D. thesis.

Jacobsen, N. G., Fuhrman, D. R. \& Fredsøe, J., 2012. A wave generation toolbox for the open-source CFD library: OpenFoam ${ }^{\circledR}$. International Journal for Numerical Methods in Fluids, 70, 1073-1088.

JP Kenny, 2009. SW Wave Hub - Metocean Design Basis. Report No. 2056. United Kingdom: Metoc plc [online]. http://platinum.vpwsys.net/ $\sim$ wavehubc/wp-content/uploads/2011/06/S18-2009.04.29-MetocDesign-Basis-Report-Rev-1-FINAL.pdf [accessed 24th January 2013].

Leijon, M., Bernhoff, H., Berg, M. and Agren, O., 2003. Economical considerations of renewable electric energy production - especially development of wave energy. Renewable Energy, 28, 1201-1209.

Leijon, M., Danielsson, O., Eriksson, M., Thorburn, K., Bernhoff, H., Isberg, J., Sundberg, J., Ivanova, I., Sjostedt, E., Agren, O., Karlsson, K. E. and Wolfbrandt, A., 2006. An electrical approach to wave energy conversion. Renewable Energy, 31, 1309-1319.

Morgan, G. C. J. Zang, J., Greaves, D., Heath, A., Whitlow, C. and Young, J., 2010. Using the rasInterFoam CFD model for wave transformation and coastal modeling. The Proceedings of the $20^{\text {th }}$ International Offshore and Polar Engineering Conference (Shanghai, China) pp. 1-9.

Smith, H. C. M., Pearce, C. and Millar, D. L., 2012. Further analysis of change in nearshore wave climate due to an offshore wave farm: An enhanced case study for the Wave Hub site. Renewable Energy, 40, 5164.

Stansby, P. K., Xu, R., Rogers, B., Hunt, A., Borthwick, A. and Taylor, P., 2008. Modelling tsunami overtopping of a sea defence by shallowwater Boussinesq, VOF and SPH methods. In: Samuels, P., Huntington, S., Allsop, W. and Harrop, J. (eds), Flood Risk management: Research and Practice. AK Leiden, The Netherlands: CRC Press/Balkema, pp 52-53.

SWERDA, 2006. South West of England Regional Development Agency: Wave Hub Development and Design Phase Coastal Processes Study Report [online]. http://www.wavehub.co.uk/wp-content/uploads/2011/ 06/Appendix-A-Coastal-Processes.pdf [accessed 14th December 2012].

Tromans, P.S., Anaturk, A. and Hagemeijer, P., 1991. A new model for the kinematics of large ocean waves - application as a design wave. The Proceedings of the $1^{\text {st }}$ International Offshore and Polar Engineering Conference (Edinburgh, UK), vol. 3, pp. 64-71.

Westphalen, J., 2010. Extreme Wave loading on offshore Wave Energy Devices using CFD. Plymouth, UK: University of Plymouth, PhD thesis.

Yemm, R. W., Henderson, R. M. and Taylor, C. A. E., 2012. The OPD Pelamis WEC: Current Status and Onward Programme [Online]. Available at: http://www.inference.phy.cam.ac.uk/sustainable/refs/Pela misStatus.pdf [accessed 23rd November 2012]. 